

\title{
Storage Device Sizing for a Hybrid Railway Traction System by Means of Bicausal Bond Graphs
}

\author{
Grace Gandanegara, Xavier Roboam, Bruno Sareni
}

\section{To cite this version:}

Grace Gandanegara, Xavier Roboam, Bruno Sareni. Storage Device Sizing for a Hybrid Railway Traction System by Means of Bicausal Bond Graphs. Proceedings of the Institution of Mechanical Engineers, Part I: Journal of Systems and Control Engineering, 2006, vol. 220, pp.269-282. 10.1243/09596518JSCE154 . hal-00777175

\section{HAL Id: hal-00777175 \\ https://hal.science/hal-00777175}

Submitted on 15 Mar 2013

HAL is a multi-disciplinary open access archive for the deposit and dissemination of scientific research documents, whether they are published or not. The documents may come from teaching and research institutions in France or abroad, or from public or private research centers.
L'archive ouverte pluridisciplinaire HAL, est destinée au dépôt et à la diffusion de documents scientifiques de niveau recherche, publiés ou non, émanant des établissements d'enseignement et de recherche français ou étrangers, des laboratoires publics ou privés. 


\title{
Storage device sizing for a hybrid railway traction system by means of bicausal bond graphs
}

G Gandanegara, X Roboam*, and B Sareni

LEEI ENSEEIHT/INP Toulouse, Toulouse, France

\begin{abstract}
In this paper, the application of bicausal bond graphs for system design in electrical engineering is emphasized. In particular, it is shown how this approach is very useful for model inversion and parameter dimensioning. To illustrate these issues, a hybrid railway traction device is considered as a case study. The synthesis of a storage device (a supercapacitor) included in this system is then discussed.
\end{abstract}

Keywords: bond graph, bicausality, model inversion, dimensioning, electrical engineering, hybrid systems

\section{INTRODUCTION}

Electrical engineering systems are more and more complex and heterogeneous, consisting of elements of different natures, strongly coupled between different physical fields. Within this framework, the system analysis becomes complicated so that a unified formalism such as the bond graph (BG) [1-4] is particularly useful. This graphical method illustrates the energetic transfers in the system. By analysing the causal bond graph, it is possible to know whether the association is physically (and energetically) convenient and then 'numerically consistent'. Homogeneous modelling and system analysis methods directly applicable for bond graphs are the major interest of bond graph formalism [2-12].

Having chosen the system architecture and parameter sizing, the system analysis process consists of verifying whether the device fulfils the requirements: this is usually done from a system's model and its simulation. On the other hand, the synthesis process consists of choosing the system structure and its sizing, directly starting from the requirements. This 'inverse process' is essential for the design of

* Corresponding author: LEEI ENSEEIHT/INP Toulouse, 2 Rue Camichel, BP 7122, Toulouse Cedex 7, 31071, France. email: Xavier.Roboam@leei.enseeiht.fr complex energetic systems and is complementary to the analysis process. Two main issues can be addressed from this approach: model inversion and parameter sizing. On bond graph formalism, the properties of bicausality can help to solve these issues [13-19].

This paper aims at showing how bond graph formalism associated with the bicausal approach can be useful in constructing inverse models and solving system design problems. A hybrid railway traction device is considered as a case study. Coupling 'energy' and 'power' sources is firstly investigated when the consumption cycles vary significantly. Indeed, the size of the energy source can be adjusted with the average power consumption, the power peaks being provided by the storage device. Furthermore, if breaking modes appear during the driving mission, it can be possible to store the breaking energy. These issues are well known for hybrid vehicles, but it has also recently appeared in railway traction.

In the paper, the example of a supercapacitor dimensioning by means of the bicausal approach is considered. To present this application, bicausality and model inversion are briefly discussed in the next section. Then, the model of a hybrid traction device is presented and the storage element dimensioning is carried out to demonstrate the interest of bicausality synthesis. Finally, conclusions are given in section 4 . 


\section{BICAUSALITY AND MODEL INVERSION}

In a causal bond graph, each bond has one causal stroke. In fact, it can be divided into two causal halfstrokes: one for the flow variable and one for the effort variable. Thus, the assignment on the model can be examined by applying this type of causality, which is called bicausality. For graphical convention, the flow information is on the bond side with a half-arrow (in the present examples, it is below the bond).

The concept of bicausality was invented and first published by Gawthrop [13]. This proposition has opened a new research field in bond graph applications [14-19].

1. System inversion. If the bond graph structure, the parameters, and the initial states are chosen and if the outputs are given from system requirements, the necessary inputs can be directly defined from the bicausal solver.

2. State estimation. If parameters, inputs, and outputs are given, the dynamic elements of initial states can be deduced. In this paper, the state estimation is not studied. Therefore the initial states of dynamic elements are considered to be known.

3. Parameter estimation. If inputs, outputs, and initial states are given and if a set of parameters are fixed, the other parameters values can be synthesized. The number of parameters that can be determined depends on the degrees of freedom in the system (number of provided inputs/outputs).

In a bicausal bond graph, the causality of each variable is separately examined. With causal halfstrokes, there are four possibilities. The conventional or physical causality is a particular case of the bicausality where both causal half-stokes are placed on the same side of the bond. Note that, if the conventional causality gives considerable information about the physical meaning of associations, the bicausality is purely conceptual and is consequently only useful for the design process.

In a bicausal bond graph, it is preferred to replace the notation Sf, Se, De, and Df by SS (source-sensor elements) [20], even if their causality is not changed (both causal half strokes are on the same side of the bond). In this case, these SS elements are called the effort source-flow sensor (for Se and Df) or the flow source-effort sensor (for $\mathbf{S f}$ and De).

An inverse model can only be obtained if the direct model is invertible. Therefore, it is necessary to firstly test this property on the bond graph before applying the bicausality inversion process. For this purpose, several definitions are employed [17].

\section{Definition 1}

Two single-input/single-output (SISO) causal paths are disjoint if they do not pass through any common variable.

\section{Definition 2}

A set $\mathbf{S}$ is disjoint if it consists of $m$ disjoint SISO causal paths.

\section{Definition 3}

The order $\omega_{p}\left(u_{i}, y_{i}\right)$ of an SISO causal path $p$ between an input $u_{i}$ and an output $y_{i}$ is defined as

$$
\omega_{p}\left(u_{i}, y_{i}\right)=n_{\mathrm{I}}(p)-n_{\mathrm{D}}(p)
$$

where $n_{\mathrm{I}}(p)$ [respectively $n_{\mathrm{D}}(p)$ ] is the number of dynamic elements in integral (respectively differential) causality crossed by this causal path.

\section{Definition 4}

The order $\omega(S)$ of a set $\mathbf{S}$ of $m$ disjoint SISO causal paths $p_{i}, i=1$ to $m$, is

$$
\omega(S)=\sum_{i=1}^{m} \omega_{p_{i}}
$$

By using these definitions on the direct bond graph, the invertibility condition for an MIMO (multi-input/multi-output) model with $m$ inputs and $m$ outputs is:

1. If there is no choice for the set of $m$ disjoint SISO causal paths, the model is structurally not invertible.

2. If there is only one choice for the set of $m$ disjoint SISO causal paths, the model is structurally invertible.

3. If there are several choices, the modified sequential causality procedure for inversion (MSCAPI) should be applied [16, 17]:

(a) Determine a set $\mathbf{S}_{0}$ whose order is the smallest among them.

(b) Replace all sources and detectors associated with the control variables or inputs and outputs by SS elements.

(c) Assign the effort source-flow source causality to the SS output elements and propagate the causal information to the SS input elements. This propagation has to arrive and impose the effort detector-flow detector causality on the input SS elements. Other elements take 
the causality due to the bicausality propagation and junction conventions. These conventions are:

- For 1 junctions:

- effort side: only one bond without a half-stroke close to the junction;

- flow side: only one bond without a halfstroke close to the junction.

- For 0 junctions:

- effort side: only one bond with a halfstroke near the junction;

- flow side: only one bond with a halfstroke near the junction.

(d) If there is at least one causality conflict, the model is not invertible. In the opposite case, it is invertible.

When the model is invertible, the following procedure can be applied to construct the inverse model (or the synthesis model) using bicausality [16].

1. Replace all source and detector elements by SS elements.

2. Apply the bicausality effort source-flow source on the output elements and the effort sensor-flow sensor on the input elements or on the elements whose parameters have to be synthesized, in relation to the degrees of freedom.

3. Propagate the bicausality from outputs to inputs. Other elements take the causality due to the bicausality propagation with respect to junction conventions.

The obtained bond graph is then called a bicausal bond graph.

\section{Model derivation}

A direct formulation with integral causality can be expressed as

$$
\frac{\mathrm{d}}{\mathrm{d} t} x=f(x, u) ; \quad y=g(x, u)
$$

Generally, some derivative causalities appear when the inversion process is applied. An implicit differential algebraic equation (DAE) model is obtained and the inverse formulation with integral (int) and derivative (der) causality is as follows

$$
\begin{aligned}
& \frac{\mathrm{d}}{\mathrm{d} t} x_{\mathrm{int}}=f_{\text {inv }}\left(x_{\mathrm{int}}, y_{\text {spec }}, \ldots, y_{\mathrm{spec}}^{n}\right) \\
& u_{\mathrm{inv}}=g_{\text {inv }}\left(x_{\text {int }}, y_{\text {spec }}, \ldots, y_{\text {spec }}^{n}\right)
\end{aligned}
$$

where $x_{\text {int }}$ is the state variable corresponding to the element that keeps an integral causality in the inverse bond graph. The specified inputs $\left(y_{\text {spec }}\right)$ of the inverse model have to be sufficiently differentiable. In particular, if the inversion is processed along an SISO causal path with a length of $n$, the corresponding input of the inverse model must be $n$ times differentiable if no symbolic manipulations are made. Some propositions have been made in reference [21] to facilitate the numerical derivation. This issue can also be solved by filtering the specified inputs of the inverse model. From a specified data file, several methods of filtering can be used with different cutoff frequencies. In particular, causal (lowpass filter) or acausal (centred weighted moving average) filtering methods can be applied. In the first case (causal) the signal phase is shifted following the cut-off frequency. In order to avoid this delay an acausal filtering can be used.

\section{APPLICATION ON A RAILWAY TRACTION DEVICE}

In order to apply these methods, a railway traction system is considered. Basically, the model of this device is a 'simplified vision' of the traction part of the Alstom BB36000 locomotive [22] (see Fig. 1). The considered structure is composed of a direct current (d.c.) voltage source, an RLC input filter feeding an induction machine that drives the mechanical

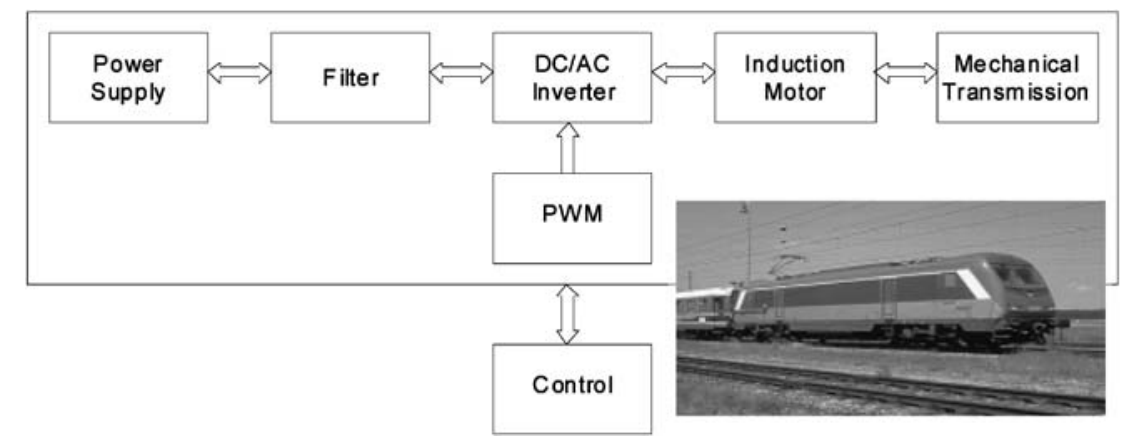

Fig. 1 The BB36000 simplified block diagram 
transmission line [22]. It has been modelled in the bond graph and several analyses have been carried out $[\mathbf{1 0 - 1 2}]$ as a model simplification or stability analysis.

In this paper, the induction motor with a d.c. motor is replaced in order to simplify the bicausal approach of the model inversion. This 'equivalent motor' is sized to present the same power balance (identical efficiency) as the one actually obtained with the induction motor. The previous voltage source inverter is then also replaced by a d.c.-d.c. chopper. The direct causal bond graph corresponding to this system is presented in Fig. 2. To test the device behaviour with real conditions, the Central Business District (CBD) cycle has been retained as the system driving mission [23]. The CBD cycle is considered as a reference for the design of traction systems. Each cycle includes a velocity acceleration phase, a constant velocity phase at $20 \mathrm{mile} / \mathrm{h}$, a velocity deceleration (or braking) phase, and a phase with zero velocity. The curves of velocity and power source applied to the present case study are illustrated in Fig. 3. It can be seen that negative powers (i.e. the regenerative phase) are obtained.

\subsection{The inverse model}

In the direct causal bond graph, the d.c. voltage source $\left(U_{\text {cont }}\right)$, the duty cycle $\alpha$ of the d.c./d.c. converter and the resistive force $\left(F_{\text {res }}\right)$ are considered as the inputs and the obtained source current and velocity as the outputs. In particular, consider the SISO causal path $L$ from the control variable $\alpha$ to the flow variable (train velocity) at the bond $42 f_{42}$ (see Fig. 2): there are four choices of SISO causal paths, where $X$ indicates that the element $X$ is crossed by the causal path:

First choice: $\omega_{1}\left(\alpha, f_{42}\right)=12$

$$
\begin{aligned}
L_{1}= & \alpha-e_{7}-e_{9}-L_{\mathrm{DC}}-f_{9}-f_{10}-e_{11}-e_{12}-I_{6} \\
& -f_{12}-f_{13}-f_{14}-f_{15}-K_{\mathrm{acc}}-e_{15}-e_{14}-e_{17} \\
& -e_{18}-I_{5}-f_{18}-f_{19}-f_{20}-f_{21}-K_{4}-e_{21} \\
& -e_{22}-e_{23}-e_{24}-I_{4}-f_{24}-f_{25}-f_{26}-f_{27} \\
& -K_{5}-e_{27}-e_{28}-e_{29}-e_{30}-I_{3}-f_{30}-f_{31} \\
& -f_{32}-f_{33}-K_{\mathrm{jac}}-e_{33}-e_{32}-e_{35}-e_{36}-I_{9} \\
& -f_{36}-f_{37}-f_{38}-K_{\mathrm{ess}}-e_{38}-e_{39}-e_{40}-I_{\mathrm{eq}} \\
& -f_{40}-f_{41}-f_{42}
\end{aligned}
$$

Second choice: $\omega_{2}\left(\alpha, f_{42}\right)=11$

$$
\begin{aligned}
L_{2}= & \alpha-e_{7}-e_{9}-L_{\mathrm{DC}}-f_{9}-f_{10}-e_{11}-e_{12}-I_{6} \\
& -f_{12}-f_{13}-f_{14}-f_{16}-C_{\mathrm{acc}}-e_{16}-e_{14}-e_{17} \\
& -e_{18}-I_{5}-f_{18}-f_{19}-f_{20}-f_{21}-K_{4}-e_{21} \\
& -e_{22}-e_{23}-e_{24}-I_{4}-f_{24}-f_{25}-f_{26}-f_{27} \\
& -K_{5}-e_{27}-e_{28}-e_{29}-e_{30}-I_{3}-f_{30}-f_{31} \\
& -f_{32}-f_{33}-K_{\mathrm{jac}}-e_{33}-e_{32}-e_{35}-e_{36}-I_{9} \\
& -f_{36}-f_{37}-f_{38}-K_{\mathrm{ess}}-e_{38}-e_{39}-e_{40}-I_{\mathrm{eq}} \\
& -f_{40}-f_{41}-f_{42}
\end{aligned}
$$

Third choice: $\omega_{3}\left(\alpha, f_{42}\right)=11$

$$
\begin{aligned}
L_{3}= & \alpha-e_{7}-e_{9}-L_{\mathrm{DC}}-f_{9}-f_{10}-e_{11}-e_{12}-I_{6} \\
& -f_{12}-f_{13}-f_{14}-f_{15}-K_{\mathrm{acc}}-e_{15}-e_{14}-e_{17} \\
& -e_{18}-I_{5}-f_{18}-f_{19}-f_{20}-f_{21}-K_{4}-e_{21} \\
& -e_{22}-e_{23}-e_{24}-I_{4}-f_{24}-f_{25}-f_{26}-f_{27} \\
& -K_{5}-e_{27}-e_{28}-e_{29}-e_{30}-I_{3}-f_{30}-f_{31} \\
& -f_{32}-f_{33}-C_{\mathrm{jac}}-e_{33}-e_{32}-e_{35}-e_{36}-I_{9} \\
& -f_{36}-f_{37}-f_{38}-K_{\mathrm{ess}}-e_{38}-e_{39}-e_{40}-I_{\mathrm{eq}} \\
& -f_{40}-f_{41}-f_{42}
\end{aligned}
$$

Fourth choice: $\omega_{4}\left(\alpha, f_{42}\right)=10$

$$
\begin{aligned}
L_{4}= & \alpha-e_{7}-e_{9}-L_{\mathrm{DC}}-f_{9}-f_{10}-e_{11}-e_{12}-I_{6} \\
& -f_{12}-f_{13}-f_{14}-f_{15}-C_{\mathrm{acc}}-e_{15}-e_{14}-e_{17} \\
& -e_{18}-I_{5}-f_{18}-f_{19}-f_{20}-f_{21}-K_{4}-e_{21} \\
& -e_{22}-e_{23}-e_{24}-I_{4}-f_{24}-f_{25}-f_{26}-f_{27} \\
& -K_{5}-e_{27}-e_{28}-e_{29}-e_{30}-I_{3}-f_{30}-f_{31} \\
& -f_{32}-f_{33}-C_{\mathrm{jac}}-e_{33}-e_{32}-e_{35}-e_{36}-I_{9} \\
& -f_{36}-f_{37}-f_{38}-K_{\mathrm{ess}}-e_{38}-e_{39}-e_{40}-I_{\mathrm{eq}} \\
& -f_{40}-f_{41}-f_{42}
\end{aligned}
$$

Given that several choices of the SISO causal path exist, it cannot be directly deduced whether the model is invertible or not. In this case, the MSCAPI procedure has to be applied. The last choice is associated with the SISO causal path with the smallest order. In this way, this path is examined. After having replaced the input (in this case, the right bond of the $\mathbf{M T F}_{\alpha}$ ) by an effort sensor-flow sensor and the output (the detector velocity) by an effort source-flow source SS element with $e=0$, the bicausality propagation does not imply any causality conflicts (see Fig. 4). Therefore, the model is invertible. The model obtained by the MSCAPI procedure is also the 


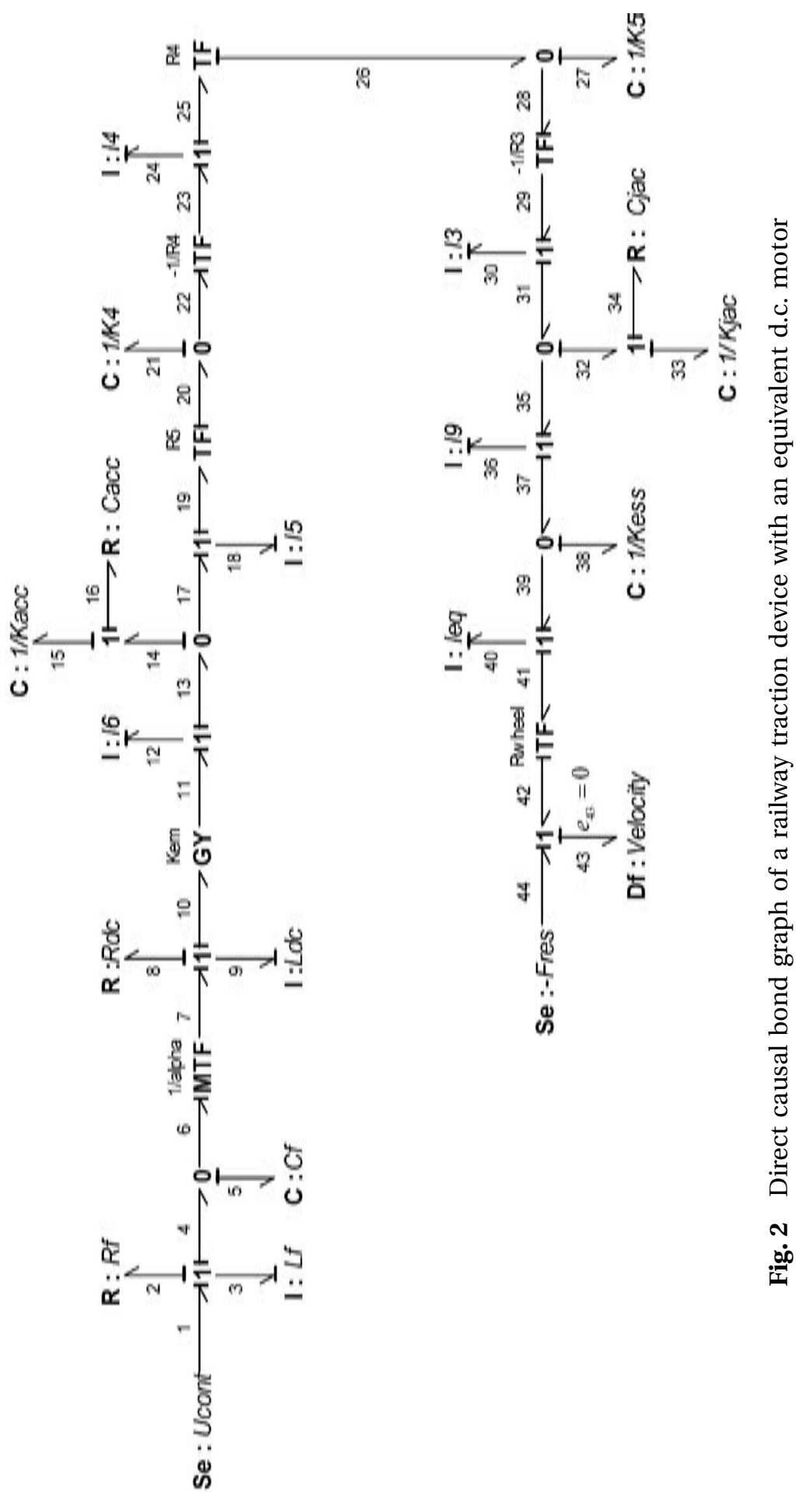




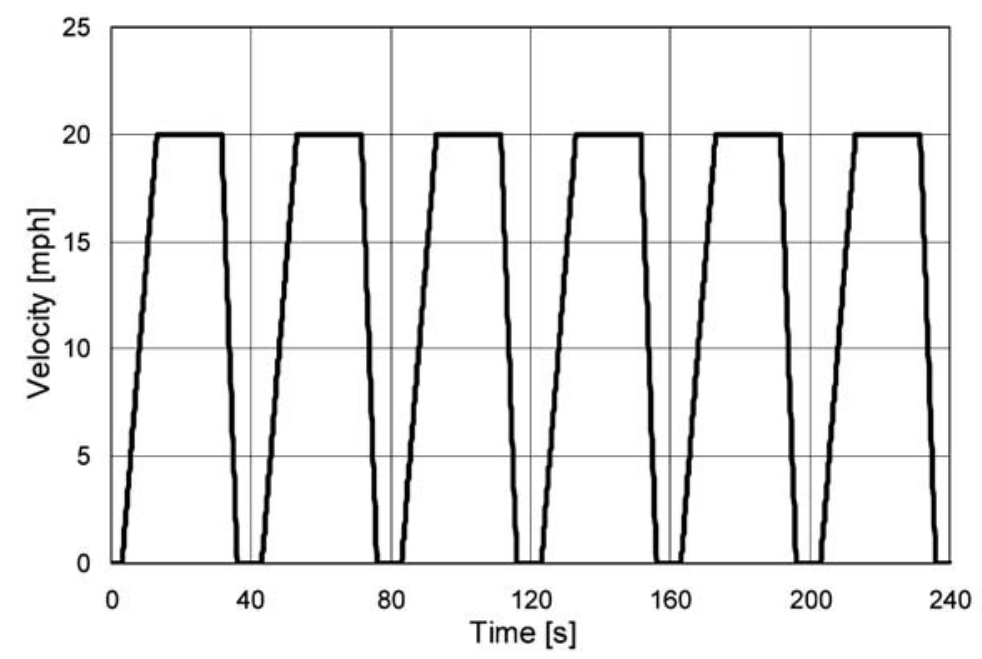

(a)



(b)

Fig. 3 CBD cycles: (a) velocity and (b) power source curves

inverse model. In this inverse model, the resistive force and the velocity information related to the CBD cycles are injected.

Note that by considering the driving mission (CBD cycles) as requirements, the electrical constraints can 'directly' be synthesized by means of this bicausal approach from the model inversion. This example emphasizes the design capacity of this methodology in electrical engineering in the context of a 'top-down' systemic approach.

Simulations are carried out by means of the $20 \mathrm{Sim}$ simulation software $[\mathbf{2 4 , 2 5}$ by using a modified library developed in the authors' laboratory, in order to take bicausality into account. In the case study, having an SISO causal path length of 10, a set of DAE is obtained for which the output (i.e. the duty cycle $\alpha$ ) is expressed versus the input (i.e the train velocity). Without any symbolic manipulation [21] of the equations, this input is differentiated 10 times in the inverse model (10 I and $\mathbf{C}$ elements are in derivative causality). Therefore, the input must be differentiable at least 10 times. The 20 Sim build-in backward differentiation formula (BDF) [18] is used as an integration method.

As a validation of the inversion process, Fig. 5 shows the duty cycle obtained from the inverse model superimposed with the one obtained from the direct model. In this particular case, the validation process consists in using the output of the direct model (i.e. the train velocity) as the input of the inverse model. Therefore the velocity is differentiable at least 10 times and is obtained in the direct model through 10 dynamic elements with integral causality. In the general case, the specified input signal must be 'sufficiently filtered' to inverse and differentiate the model. Some symbolic manipulations of the derived model can also be made, as given in reference [21]. Note that this includes the control strategy, contrary to the inverse model. This result proves that the inverse model is validated. 


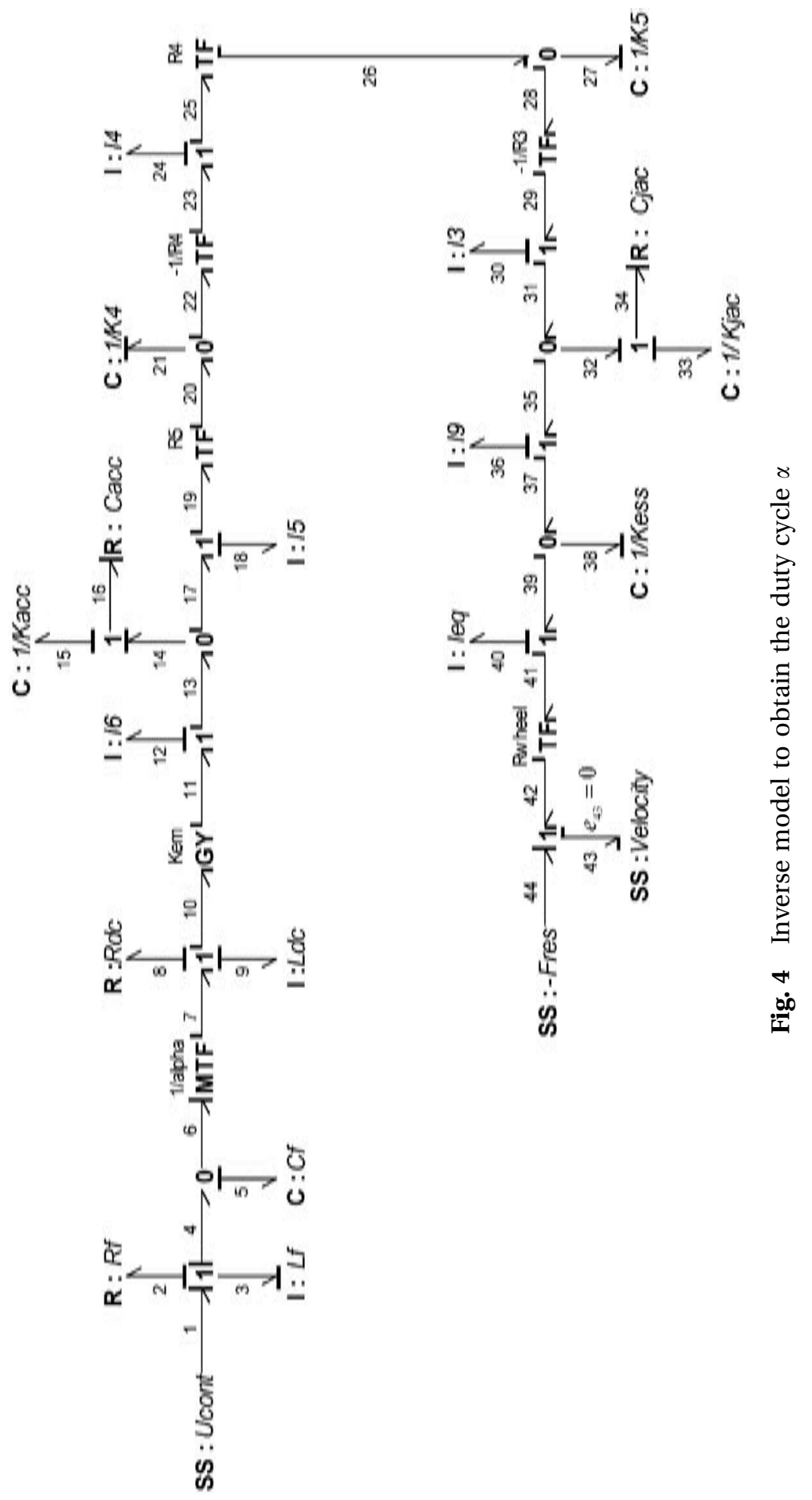






Fig. 5 Comparison between the direct model and inverse model responses: duty cycle $\alpha$

\subsection{Bicausal synthesis of the storage element in a hybrid system}

\subsubsection{Hybrid systems}

In numerous devices, such as the one considered here as a case study, the loading power is very timevariable (see here the CBD cycle of Fig. 3) so that the peak power is far from the average consumed power. For such systems, the main source must be 'oversized' to take into account the maximum peak power demand, which presents a great drawback from an economic point of view. The general idea in hybrid systems is well known for electrical vehicles but it also becomes applicable in other fields. For railway traction systems, hybridization can offer advantages such as a reduction of energy consumption and of pollution (carbon emissions) when diesel-electric devices are used.

In hybrid systems, it is necessary to associate the main energy source with a storage device, such as supercapacitors, batteries of accumulators, or inertia wheels. With such components, the primary energy source will only have to furnish the average loading power and the average system losses. The variations of the consumed power can be provided by the storage element(s). In this type of system, the concept of energy sources is used for the sources supplying the average loading power and power sources for those furnishing the loading power variations in short periods according to the driving mission time.

\subsubsection{Energy management and storage control}

A supercapacitor will be considered here as the storage element. This device is modelled by a low resistance (representing storage losses) in series with an important capacitance. Even if this model is rather simplified, it is energetically significant and sufficiently accurate to demonstrate the synthesis process. This storage element is connected to the primary bus with a d.c./d.c. boost converter including the 'boost inductance'. The storage current $\left(I_{\text {store }}\right)$ is then controllable (see Fig. 6).

The proposed energy management strategy is the following.

1. For a given and nearly constant d.c. bus voltage $\left(V_{\text {bus }}\right)$, the loading power variations are equivalent to the loading current variations ( $\left.I_{\text {load }}\right)$.

2. A low pass filtering (LPF) of the loading current is considered, the idea being to control the system in such a way that the primary energy source only provides the lowpass filtered loading currents $\left(I_{\text {load }}^{\mathrm{LPF}}\right)$. As a result, the primary source only provides the sum of the average loading power, the average value of the system losses, and the lowfrequency power harmonics. On the contrary, the storage device must provide the high-frequency loading currents $\left(I_{\text {load }}^{\mathrm{HF}}\right)$. The bandwidth of the LPF is determined according to the primary source specifications.

3. The system is controlled to ensure that the storage element provides the high-frequency (HF) part of the loading current. Note in this case that the average power produced by the storage element is null on a time interval superior to the inverse of the filtering cut-off frequency. In this way, the loading state of the storage device remains almost constant: the loading management of the storage element is therefore really simplified. 


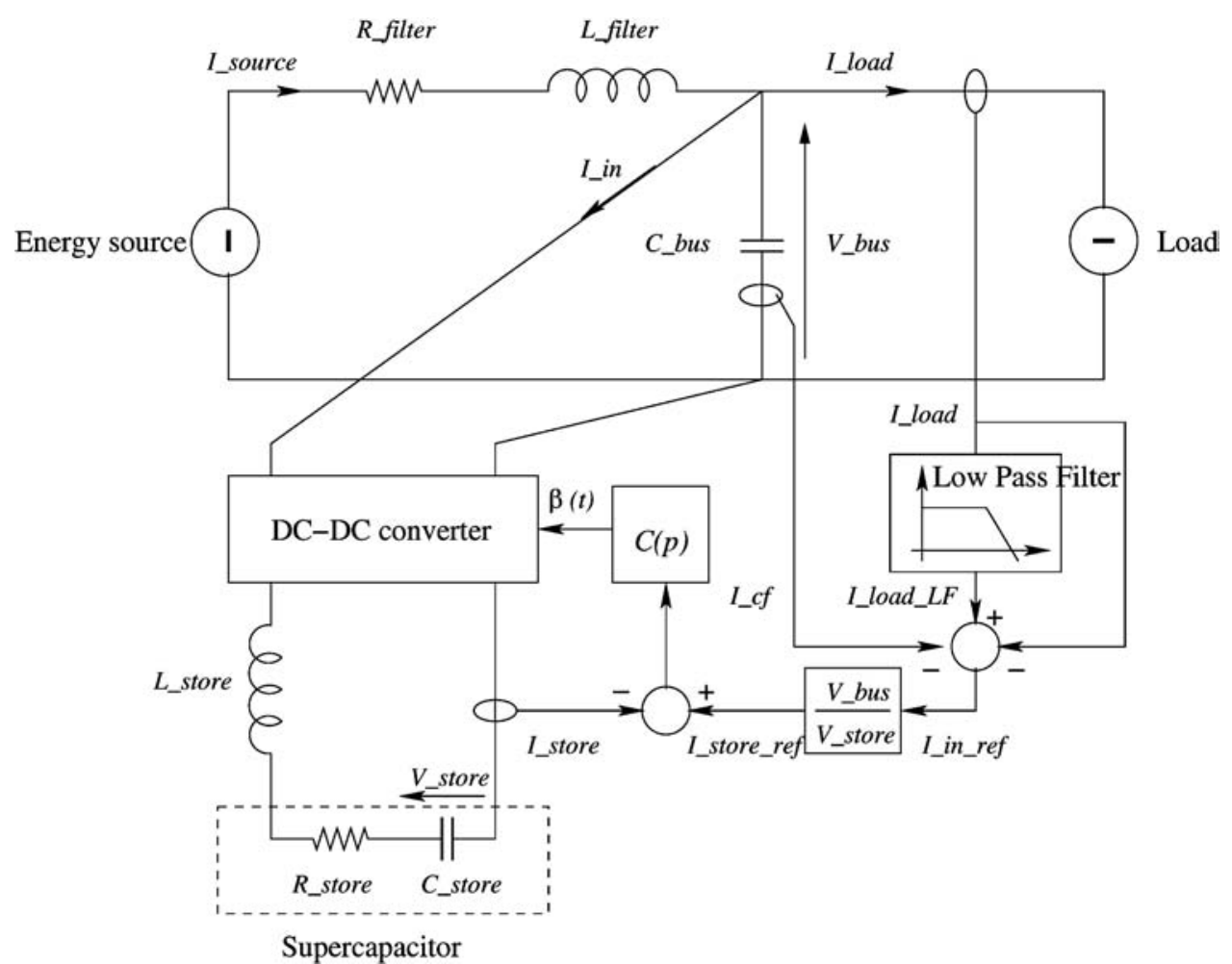

Fig. 6 Synoptic of a hybrid system with a storage element

In Fig. 3(b) the maximum loading power is $1 \mathrm{MW}$. An energy source needs to be applied that is sized up to $400 \mathrm{~kW}$ ( 40 per cent of the maximum loading power). This reduction value can be obtained by tuning the filter bandwidth. To accomplish the objective, a second-order filter is applied with a bandwidth (cut-off frequency) of $0.01 \mathrm{~Hz}$. This frequency is also convenient for filtering the power variations of the considered system with the CBD cycles.

The control of the storage element is defined in Fig. 7. The storage element should provide the HF part of the consumed current $\left(I_{\text {load }}\right)$. Respecting the power transfer orientation gives

$$
I_{\text {source }}=I_{\text {cf }}+I_{\text {in }}+I_{\text {load }}
$$

where

$$
\begin{aligned}
& I_{\mathrm{source}} \approx I_{\mathrm{load}}^{\mathrm{LPF}} \\
& I_{\mathrm{cf}} \approx 0
\end{aligned}
$$

The HF current $I_{\text {in }}$ is indirectly set by controlling the storage current $I_{\text {store }}$ filtered by the boost inductance from the duty cycle of the d.c./d.c. converter. A PI controller is used to control $I_{\text {store }}$ in order to comply with the desired specifications for $I_{\mathrm{in}}$. The parameter values are determined as

$$
C(p)=K\left(1+\frac{1}{T_{i} p}\right)
$$

with

$$
T_{i}=\frac{L_{\text {store }}}{R_{\text {store }}} \quad \text { and } \quad K=\frac{L_{\text {store }}}{T_{\mathrm{CL}}}
$$

where $T_{\mathrm{CL}}$ is the desired closed-loop time constant.

\subsubsection{Storage synthesis}

In the particular case of a supercapacitor, an analytical approach is possible to synthesize the $C_{\text {store }}$ value. This analytical process is used as a comparison with the bicausal approach which is the main aim of this paper.

Analytical approach. The energy that can be stored or supplied by the storage element is defined as

$$
E_{\text {store }}(t)=\int_{0}^{t}\left[V_{\text {bus }}(\tau) I_{\text {in }}(\tau)\right] \mathrm{d} \tau+E_{\text {store }}(0)
$$

The stored energy of the supercapacitor $E_{\mathrm{C}}$ is

$$
E_{\mathrm{C}}(t)=\frac{1}{2} C\left[V_{\text {store }}(t)\right]^{2}=\frac{1}{2} C\left[\beta(t) V_{\text {bus }}(t)\right]^{2}
$$




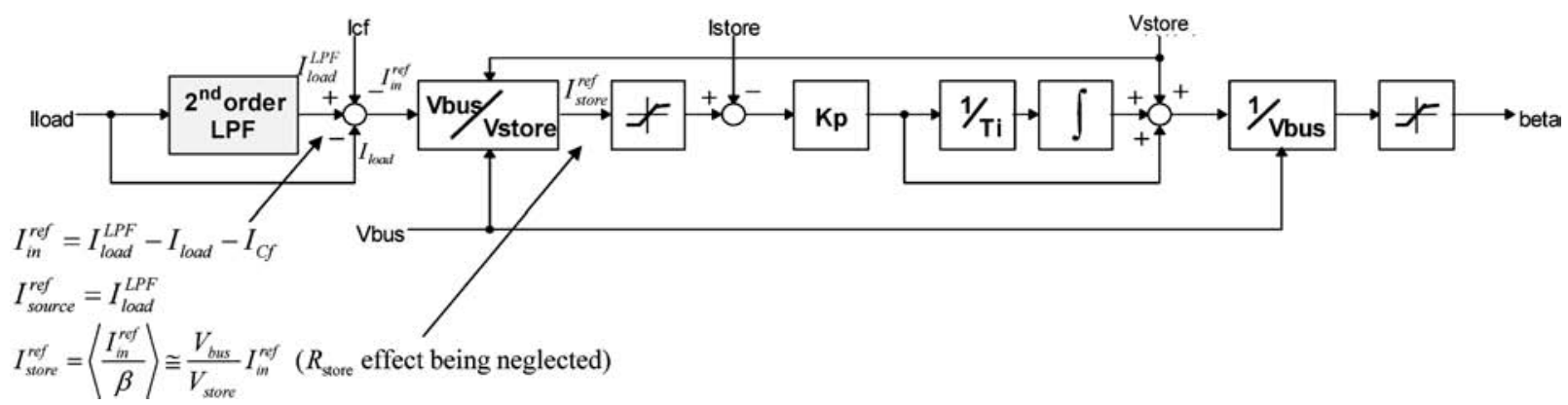

Fig. 7 Schema of the storage controller by HF filtering

In fact, $E_{\text {store }}$ and $E_{\mathrm{C}}$ are almost equivalent if the chopper and the resistance $R_{\text {store }}$ losses are neglected (the average stored energy in the inductance $L_{\text {store }}$ is also zero)

$$
E_{\text {store }}(t) \approx E_{\mathrm{C}}(t)
$$

\section{First assumption}

If the bus voltage is constant, then

$$
E(t)=V_{\text {bus }} \int_{0}^{t} I_{\text {in }}(\tau) \mathrm{d} \tau+E_{\text {store }}(0)
$$

\section{Second assumption}

If the discharge state of the supercapacitor is 50 per cent of the nominal voltage, there are two extreme cases.

1. The case where $V_{\text {store }}$ is maximal (100 per cent):

$$
\max \left(V_{\text {store }}\right)=V_{\text {bus }} \text { when } \beta_{\max }=1
$$

The associated energy is

$$
E_{\max }=\frac{1}{2} C\left(V_{\text {bus }}\right)^{2}
$$

2. The case where $V_{\text {store }}$ is minimal (50 per cent):

$$
\min \left(V_{\text {store }}\right)=0.5 V_{\text {bus }} \text { when } \beta_{\min }=0.5
$$

The associated energy is

$$
E_{\min }=\frac{1}{2} C\left(\frac{V_{\text {bus }}}{2}\right)^{2}=\frac{1}{8} C\left(V_{\text {bus }}\right)^{2}
$$

To generalize, if the discharge depth is $x \%$, then the minimal energy will be

$$
E_{\min }=\frac{1}{2} C\left(x\{\%\} V_{\text {bus }}\right)^{2}
$$

From these assumptions,

$$
\begin{aligned}
\frac{E_{\text {max }}-E_{\min }}{V_{\text {bus }}}= & \frac{3}{8} C V_{\text {bus }} \approx \max \left[\int_{0}^{t} I_{\text {in }}(\tau) \mathrm{d} \tau\right] \\
& -\min \left[\int_{0}^{t} I_{\text {in }}(\tau) \mathrm{d} \tau\right]
\end{aligned}
$$

The current profile can be directly obtained from the relation

$$
I_{\text {in }}=I_{\text {load }}^{\mathrm{LPF}}-I_{\text {load }}=I_{\text {load }}^{\mathrm{HF}}
$$

Finally, the minimal value of the supercapacitor $C_{\text {store }}$ can be calculated as

$$
C_{\text {min }}=\frac{8}{3 V_{\text {bus }}}\left(\max \left(\int_{0}^{t} I_{\text {in }}(\tau) \mathrm{d} \tau\right)-\min \left(\int_{0}^{t} I_{\text {in }}(\tau) \mathrm{d} \tau\right)\right)
$$

Bicausal approach. Bicausality is another way to determine this supercapacitor value. Both voltage $\left(V_{\text {bus }}\right)$ and current $\left(I_{\text {in }}\right)$ should be provided by the storage element and can easily be deduced from the bicausal bond graph of Fig. 8. In this BG, the loading current (SS element) has been synthesized from the inverse BG model developed in section 3.1. For this purpose, the train velocity has been filtered with a causal filtering so that the inverse system can be derived with the BDF algorithm of 20 Sim. From the inverse model presented in Fig. 4 , the current $f_{6}$ and the associated voltage $e_{6}$ can be obtained at the output of the LRC filter (see Fig. 9). It can be seen that the voltage of the input filter is almost constant. Therefore, the power can be tuned if the current is itself controlled.

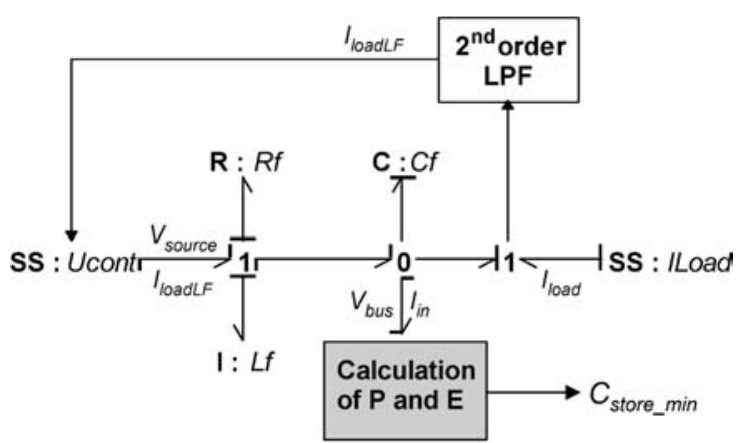

Fig. 8 Inverse model with a submodel to calculate the minimal value of $C_{\text {store }}$ 


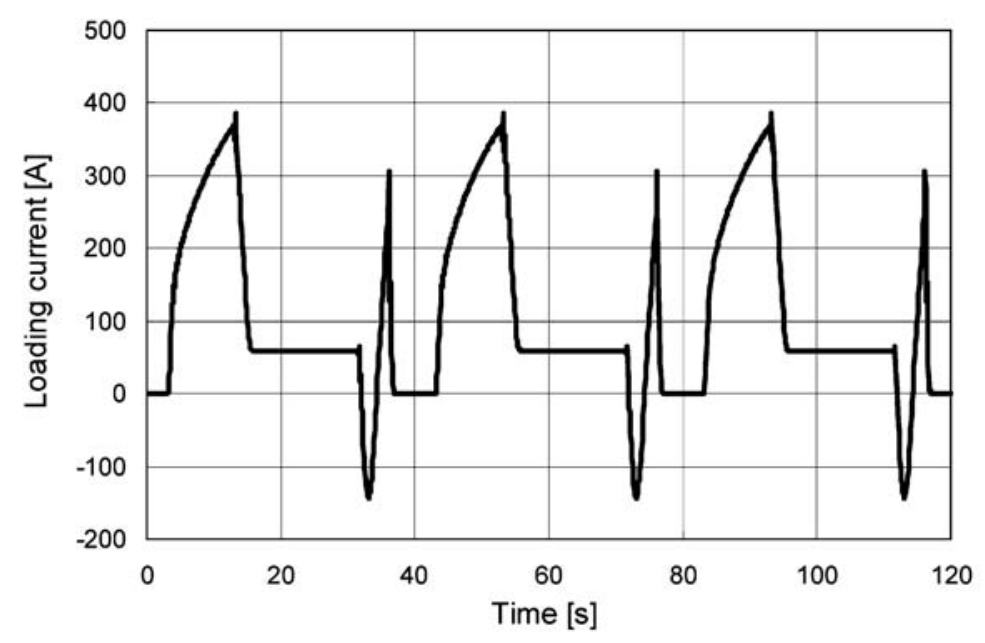

(a)

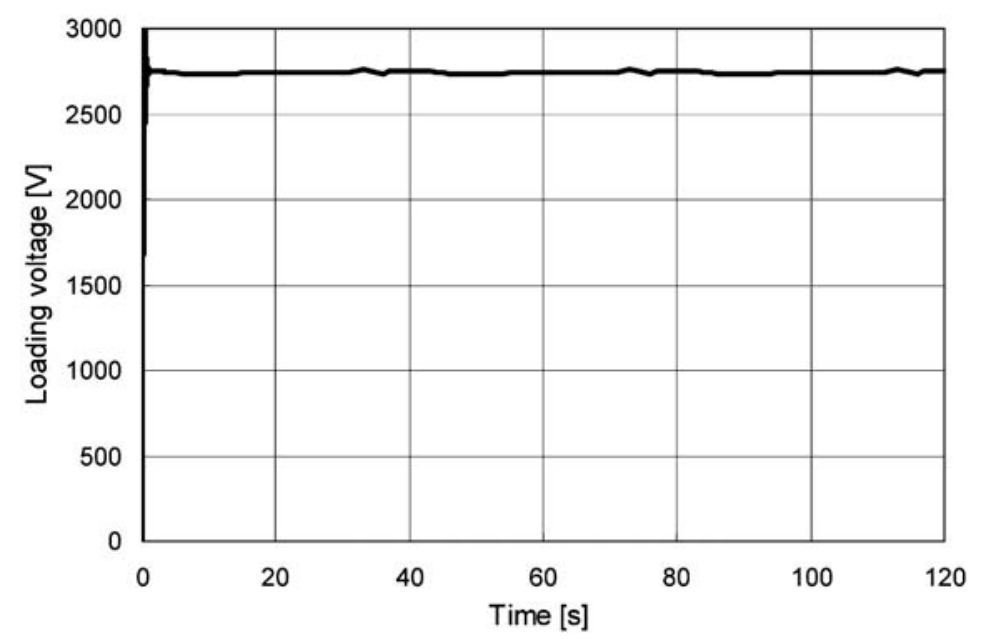

(b)

Fig. 9 Loading current and voltage for CBD cycles

The bicausal BG of Fig. 4 allows the direct calculation of the stored energy to be made at any given time. It is sufficient to take the maximal and minimal values of the stored energy during the CBD cycles. For a 50 per cent discharge depth, the supercapacitor value is obtained using the following formula

$$
C_{\text {min }}=\frac{8}{3\left(V_{\text {bus }}\right)^{2}}\left(E_{\max }-E_{\min }\right)
$$

The general expression for a discharge depth of $x \%$ is

$$
C_{\min }=\frac{2}{(1-x\{\%\})^{2}\left(V_{\text {bus }}\right)^{2}}\left(E_{\max }-E_{\min }\right)
$$

Note that the obtained minimal value $C_{\min }$ is only valid for a certain driving mission (here the CBD cycle). If the power profile is changed, this value should be recalculated.

With the CBD cycles (cf. Fig. 3), the loading power is extremely time variable (from $-400 \mathrm{~kW}$ to $1 \mathrm{MW}$ ). For this kind of application, a hybrid structure is appropriate and it is interesting to integrate a storage element.

Numerical applications. Applying both methods previously presented with a discharge depth of 50 per cent gives:

With the analytical approach

$$
\max \left(\int_{0}^{t} I_{\text {in }}(\tau) \mathrm{d} \tau\right)-\min \left(\int_{0}^{t} I_{\text {in }}(\tau) \mathrm{d} \tau\right)=2081.514
$$

Then

$$
C_{\min }=\frac{8}{3} \frac{2081.514}{2750}=2.018 \mathrm{~F}
$$

With the bicausality approach

$$
C_{\text {min }}=2.022 \mathrm{~F}
$$

Note that these methods give quite similar results. The difference is related to the accuracy of both integration methods. 


\subsection{Storage synthesis validation}

In order to validate the bicausal approach based on storage synthesis, the direct model of Fig. 6 is simulated with the loading current illustrated in
Fig. 9(a). The storage element is represented by a supercapacitor of $C_{\text {store }}=3 \mathrm{~F}$ with internal resistive losses $R_{\text {store }}=5 \mathrm{~m} \Omega$. The boost inductance is fixed at $L_{\text {store }}=1 \mathrm{mH}$ in order to smooth the current ripple correctly. The responses of this direct BG model are

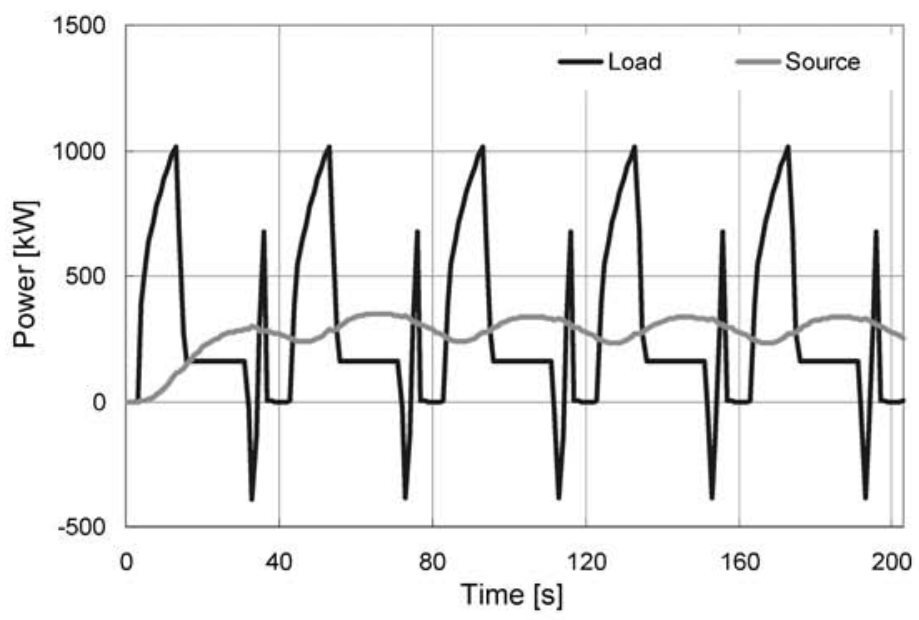

(a)

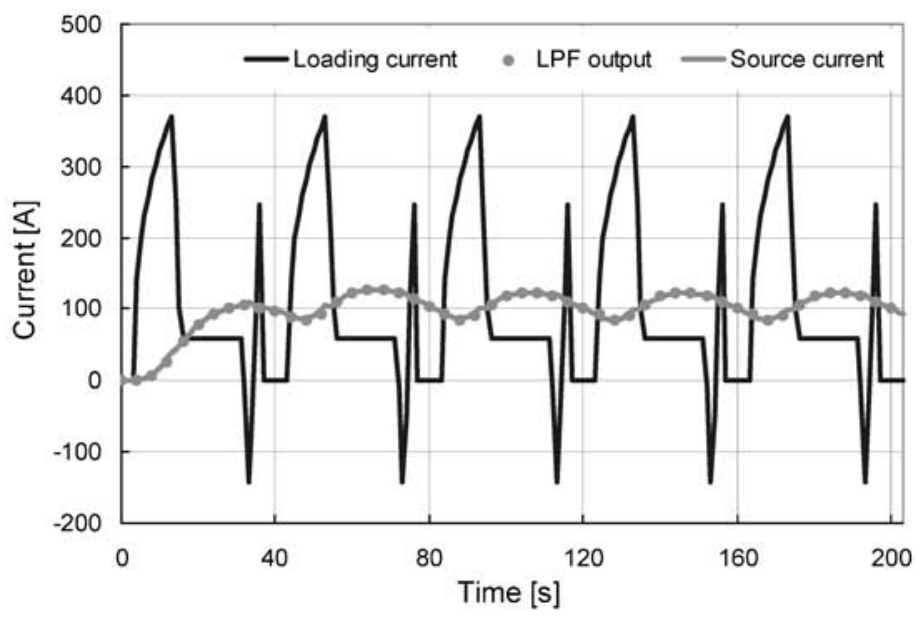

(b)

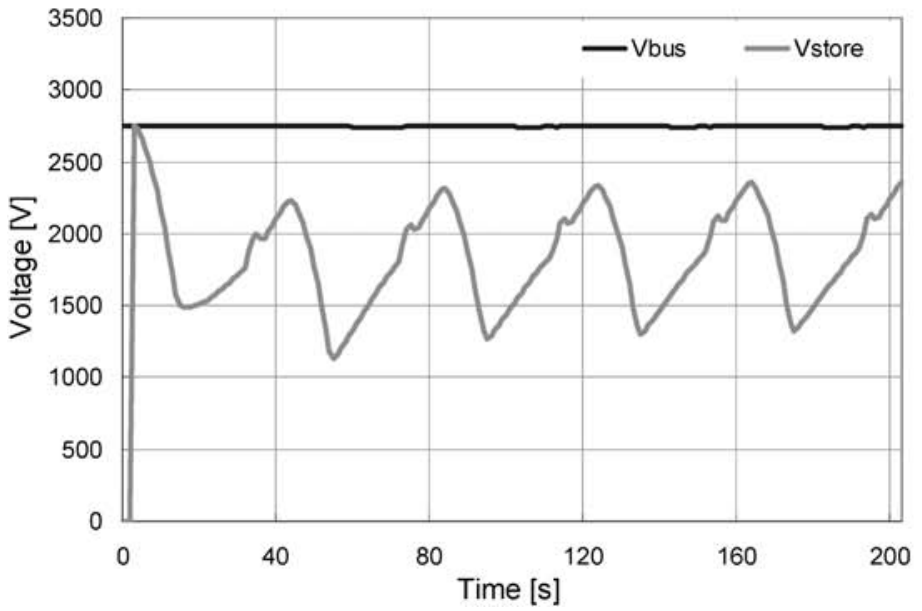

(c)

Fig. 10 Analysis of the entire hybrid system modelled by the direct causal bond graph: (a) loading and primary source powers; (b) loading, LPF, and source currents; (c) bus and $C_{\text {store }}$ voltages 
presented in Fig. 10. It can be seen that the power provided by the primary source is smoothed. This source only provides the low-frequency (LF) part of the loading current. On the curve of Fig. 10(b), the primary source current is perfectly superimposed with the current filtered by the LPF, which shows that the energy management is perfectly processed. The curve of Fig. 10(c) allows verification that the 50 per cent maximum of the discharge depth is actually obtained.

Note that for this driving cycle, the source can be sized according to a loading power of $350 \mathrm{~kW}$ instead of $1 \mathrm{MW}$, which is about 35 per cent of the maximal loading power.

\section{CONCLUSIONS}

In this paper, the efficiency of the bicausal approach for system design in electrical engineering has been emphasized, mainly with the aim of model inversion and parameter sizing. This 'synthetic approach' has been applied to a typical electrical engineering device: a hybrid railway traction system.

First, the capacity of the bicausality for model inversion has been put forward. Note that by considering a given driving mission as the requirement, the input constraints can 'directly' be synthesized by means of this bicausal approach, contrary to the classical 'analytical approach', which usually needs several iteration cycles between allocations (design choices) and analysis (allocation verification).

Second, the bicausality has been used to synthesize a storage element in the wide application case of hybrid systems. For such systems, the idea is to size the primary energy source following average power consumption. For this purpose, a storage element, here a supercapacitor, is added in order to provide the intermittent power. By using bicausality, the profile required for the storage power can be directly deduced and a value of the supercapacitor can be efficiently synthesized following the choice of the discharge depth. Note that the minimal value of the supercapacitor is only valid for the considered mission cycle ensuring that the finality character (i.e. the actual functioning mode of the system) is directly linked with the design process, as for any systemic approach.

More generally, this application example has emphasized the capacity of the bicausal methodology with a view to a systemic approach using electrical engineering design.

\section{REFERENCES}

1 Paynter, H. Analysis and design of engineering systems, 1961 (MIT Press, Boston, Massachusetts).

2 Karnopp, D., Margolis, D., and Rosenberg, R. System dynamics: modelling and simulation of mechatronic systems, 3rd edition, 2000 (John Wiley \& Sons, Inc., New York).

3 Gawthrop, P. J. and Smith, L. P. S. Metamodelling: bond graphs and dynamic systems, 1996 (PrenticeHall, Hemel Hempstead, Hertfordshire).

4 Dauphin-Tanguy, G. Les bond graphs, 2000 (Hermès, Paris).

5 Sueur, C. and Dauphin-Tanguy, G. Bond-graph approach for structural analysis of MIMO linear systems. J. Franklin Inst., 1991, 328, 55-70 (Pergamon Press).

6 Dauphin-Tanguy, G., Rahmani, A., and Sueur, C. Bond graph aided design of controlled systems. Simulation Practice and Theory, 1999, 7, 493-513 (Elsevier Science BV).

7 Sueur, C. and Dauphin-Tanguy, G. Bond graph approach to multi-time scale systems analysis. J. Franklin Inst., 1991, 328, 1005-1026 (Pergamon Press).

8 Louca, L. S., Stein, J. L., and Hulbert, G. M. A physical-based model reduction metric with an application to vehicle dynamics. In Proceedings of the 4th IFAC Symposium on Nonlinear control systems, Enschede, The Netherlands, 1998.

9 Louca, L. S. and Stein, J. L. Energy-based model reduction of linear systems. In Proceedings of the International Conference on Bond graph modeling and simulation (ICBGM'99), vol. 31, San Francisco, California, 1999 (Society for Computer Simulation).

10 Gandanegara, G., Sareni, B., Roboam, X., and Dauphin-Tanguy, G. Bond graph multi-time scale analysis of a railway traction system. In Proceedings of the 9th European Conference on Power electronics and applications (EPE'01), Graz, Austria, September 2001.

11 Gandanegara, G., Roboam, X., Sareni, B., and Dauphin-Tanguy, G. Modeling and multi-time scale analysis of railway traction systems using bond graphs. In Proceedings of the International Conference on Bond graph modeling and simulation (ICBGM'01), vol. 33, no. 1, Phoenix, Arizona, January 2001, pp. 62-67 (Society for Computer Simulation).

12 Gandanegara, G., Roboam, X., Sareni, B., and Dauphin-Tanguy, G. One model for one frequency range: comparison of bond graph based simplification methods. In Proceedings of the International Conference on Bond graph modeling and simulation (ICBGM'03), vol. 35, no. 2, Orlando, Florida, January 2003, pp. 53-58 (Society for Computer Simulation).

13 Gawthrop, P. J. Bicausal bond graphs. In Proceedings of the International Conference on Bond graph modeling and simulation 1995 (ICBGM'95), Las Vegas, USA, vol. 27, January 1995, pp. 83-88 (Society for Computer Simulation). 
14 Gawthrop, P. J. Physical interpretation of inverse dynamics using bicausal bond graphs. J. Franklin Inst., 2000, 337, 743-769 (Elsevier Science Ltd.).

15 Mechin, O., Marquis-Favre, W., Scavarda, S., and Ferbach, P. Sizing of an electric power steering system on dynamic and energetic criteria. In Proceedings of the International Conference on Bond graph modeling and simulation (ICBGM'03), vol. 35, no. 2, Orlando, Florida, January 2003, pp. 137-147 (Society for Computer Simulation).

16 Ngwompo, R. F., Scavarda, S., and Thomasset, D. Inversion of linear time-invariant SISO systems modelled by bond graph. J. Franklin Inst., 1996, 333, 157-174 (Elsevier Science Ltd).

17 Ngwompo, R. F. Contribution au dimensionnement des systèmes sur des critères dynamiques et énergétiques. PhD Thesis, INSA de Lyon, 1997.

18 Ngwompo, R. F. and Gawthrop, P. J. Bond graphbased simulation of non-linear inverse systems using physical performance specifications. J. Franklin Inst., 1999, 336, 1225-1247 (Elsevier Science Ltd).

19 Ngwompo, R. F. and Scavarda, S. Dimensioning problems in system design using bicausal bond graphs. Simulation Practice and Theory, 1999, 7, 577-587 (Elsevier Science BV).

20 Gawthrop, P. J. and Smith, L. Causal augmentation of bond graphs with algebraic loops. J. Franklin Inst., 1992, 329(2), 291-303 (Elsevier Science Ltd).

21 van Dijk, J. and Breedveld, P. C. Simulation of system models containing zero-order causal path - I. Classification of zero-order causal paths. J. Franklin Inst., 1991, 328(5/6), 959-979 (Elsevier Science Ltd).

22 Lochot, C., Roboam, X., de Fornel, B., and Moll, F. High speed railway traction system modelling for simulating electromechanical interactions. In Proceedings of the World Conference on Railway research (WCRR'97), Firenze, Italy, November 1997.

23 Emission test cycles: Central Business District (CBD), Ecopoint Inc., URL: http://www.dieselnet.com/ standards/cycles/cbd.html.

24 20-Sim, Controllab Products BV, URL: http:// www.20sim.com

25 Broenink, J. F. and Kleijn, C. Computer-aided design of mechatronic systems using 20-Sim 3.0. In Proceedings of the 2nd Workshop on European scientific and industrial collaboration (WESIC'99), Newport, UK, 1999, pp. 27-34.

\section{APPENDIX}

Notation

C

bond graph generalized capacitancetype storage element

$C(p) \quad$ controller transfer function with $p$ as the Laplace parameter

De (or Df) bond graph effort (or flow) detector element

$e$

$f$

GY

I

bond graph generalized effort

variable

bond graph generalized flow

variable

bond graph gyrator element

bond graph generalized inertia-type storage element

$I_{\text {cf }}$

$I_{\text {in }}$

$I_{\text {load }}$

$I_{\text {load }}^{\text {HF }}$

$I_{\text {load }}^{\mathrm{LPF}}$

$I_{\text {load }}$

$I_{\text {source }}$

$I_{\text {store }}$

$\mathbf{R}$

filter (bus) capacitor current

input current of the storage

converter element

loading current

high-frequency loading current

lowpass filtered loading current

main source current

supercapacitor current

bond graph generalized dissipated

element

Se (or Sf) bond graph effort (or flow) source

element

SS

generic bond graph source-sensor

element

TF

$V_{\text {bus }}$

$V_{\text {store }}$

$\langle x\rangle$

bond graph transformer element

bus voltage

supercapacitor voltage

average value of $x(t)$

$\alpha$ or $\beta \quad$ converter duty cycle

0 bond graph common effort junction

1 bond graph common flow junction 